\title{
Evaluation of efficacy and safety markers in a phase II study of metastatic colorectal cancer treated with aflibercept in the first-line setting
}

Diether Lambrechts ${ }^{*}, 1,2,12$, Bernard Thienpont ${ }^{1,2,12}$, Vincent Thuillier ${ }^{3}$, Xavier Sagaert ${ }^{4}$, Matthieu Moisse ${ }^{1,2}$, Gilian Peuteman ${ }^{1,2}$, Carles Pericay ${ }^{5}$, Gunnar Folprecht ${ }^{6}$, John Zalcberg ${ }^{7}$, Chiara Zilocchi ${ }^{8}$, Emmanuelle Margherini ${ }^{9}$, Marielle Chiron $^{10}$ and Eric Van Cutsem ${ }^{11}$

${ }^{1}$ Vesalius Research Center, VIB, Leuven, Belgium; ${ }^{2}$ Department of Oncology, Laboratory for Translational Genetics, KU Leuven, Leuven, Belgium; ${ }^{3}$ Department of Biostatistics, Sanofi, Chilly-Mazarin, France; ${ }^{4}$ Department of Pathology, KU Leuven, Leuven, Belgium; ${ }^{5}$ Hospital de Sabadell, Corporació Sanitaria Parc, Taulí-Institut Universitari, Sabadell, Barcelona, Spain; ${ }^{6}$ Universitätsklinikum Carl Gustav Carus, Dresden, Germany; ${ }^{7}$ School of Public Health and Preventive Medicine, Monash University, Melbourne, USA; ${ }^{8}$ Sanofi, Milan, Italy; ${ }^{9}$ Sanofi, Oncology Division, Vitry-sur-Seine, France; ${ }^{10}$ Translational and Experimental Medicine, Oncology, Sanofi, Vitry-sur-Seine, France and ${ }^{11}$ Digestive Oncology, University Hospitals Leuven and KU Leuven, Leuven, Belgium

Background: Aflibercept (ziv-aflibercept) is an anti-angiogenic agent recently approved in combination with FOLFIRI for the treatment of metastatic colorectal cancer $(\mathrm{mCRC})$ patients previously treated with oxaliplatin. Despite heterogeneity in response to aflibercept, no biomarkers for efficacy or adverse effects have been identified. Here we present biomarker data from the randomised phase II AFFIRM trial assessing aflibercept in combination with mFOLFOX6 first line in mCRC.

Methods: Ninety-six somatic mutations in key oncogenic drivers of mCRC and 133 common single-nucleotide polymorphisms (SNPs) in vascular endothelial growth factor (VEGF) pathway genes were analysed, and 27 plasma markers measured at baseline, during and after treatment. We assessed correlations of these three classes of biomarkers with progression-free survival (PFS) and adverse events (AEs).

Results: Somatic mutations identified in KRAS, BRAF, NRAS, PIK3CA and PIK3R1 did not significantly correlate with PFS (multiple testing-adjusted false discovery rate (FDR) or multiple testing-adjusted FDR $>0.3$ ). None of the individual SNPs correlated with PFS (multiple testing-adjusted FDR $>0.22$ ), but at the gene level variability in VEGFB significantly correlated with PFS (multiple testing-adjusted FDR $=0.0423$ ). Although none of the plasma markers measured at baseline significantly correlated with PFS, high levels of circulating IL8 at baseline together with increased levels of IL8 during treatment were significantly associated with reduced PFS (multiple testing-adjusted FDR $=0.0478$ ). No association was found between biomarkers and AEs.

Conclusions: This represents the first biomarker study in mCRC treated with aflibercept. High IL8 plasma levels at baseline and subsequent increases in IL8 were associated with worse PFS, suggesting that IL8 may act as a potentially predictive biomarker of aflibercept treatment outcome.

\footnotetext{
*Correspondence: Dr D Lambrechts; E-mail: Diether.Lambrechts@vib-kuleuven.be.

${ }^{12}$ These authors contributed equally to this work.
}

Received 21 March 2014; revised 27 July 2015; accepted 4 August 2015; published online 10 September 2015 
Angiogenesis inhibitors have been established over the past decade as valuable tools to inhibit tumour growth and improve tumour response to chemotherapy. These include the humanised monoclonal antibody against vascular endothelial growth factor (VEGFA), bevacizumab (Avastin; Bennouna et al, 2013), and the soluble humanised chimeric VEGF receptor fusion protein, aflibercept (ziv-aflibercept; Van Cutsem et al, 2012; Chiron et al, 2014). Despite the significantly prolonged survival observed with these angiogenesis inhibitors, patients still succumb to their disease, suggesting that inhibition of a single-angiogenic growth factor alone is insufficient to induce vessel normalisation and stop tumour growth. Indeed, subsets of patients treated with antiangiogenic therapies do not respond, gradually develop resistance or present with therapy-related adverse events (AEs) (Jubb and Harris, 2010). The identification of potential biomarkers that predict response to anti-angiogenic treatment (Lambrechts et al, 2013) or the occurrence of these AEs is thus warranted, as it will enable better patient selection for anti-angiogenic therapy (Lambrechts et al, 2014; Schneider et al, 2014).

Aflibercept is an anti-angiogenic agent, which has recently been approved in combination with FOLFIRI for the treatment of patients with metastatic colorectal cancer (mCRC) who were previously treated with oxaliplatin. The approval was based on data obtained from the VELOUR trial-a multicenter, randomised, placebo-controlled phase III trial, which compared the efficacy of aflibercept $v s$ placebo in combination with the FOLFIRI regimen as a second-line treatment for patients with mCRC previously treated with oxaliplatin (Van Cutsem et al, 2012; Joulain et al, 2013). AFFIRM, an open-label, non-comparative, phase II study, was conducted to assess the combination of aflibercept and modified FOLFOX6 (mFOLFOX6) given as first-line therapy in patients with mCRC (Pericay et al, 2012). The primary end point was 12-month progression-free survival (PFS), whereas exploration of biomarkers was among the secondary objectives.

In an attempt to understand the key factors associated with aflibercept efficacy and safety, we conducted an investigation assessing biomarkers for aflibercept treatment in prospectively collected tumour tissues and serially sampled plasma from patients participating in the AFFIRM study. Plasma proteins and genetic variants, representing either single-nucleotide polymorphisms (SNPs) in VEGF pathway genes or somatic mutations in key oncogenic drivers of mCRC, were analysed to assess if they could predict response to aflibercept with respect to PFS. Subsequently, we also assessed whether any of these markers correlated with antiangiogenic drug-induced AEs, such as gastrointestinal perforation, thrombosis, hypertension and proteinuria.

\section{MATERIALS AND METHODS}

Patients. The intent-to-treat population comprised 236 randomised patients with mCRC randomised to receive either mFOLFOX6 plus $4 \mathrm{mg} \mathrm{kg}^{-1}$ IV aflibercept every 2 weeks $(N=119)$ or mFOLFOX6 alone $(N=117)$. Patient eligibility criteria have been previously described (Pericay et al, 2012). The primary end point of the study was to estimate PFS at 12 months. Secondary objectives included the objective overall response rate, $\mathrm{PFS}$, the overall survival (OS) time and safety profiling (Ciombor et al, 2013). Response was recorded based on Response Evaluation Criteria in Solid Tumors (RECIST, version 1.0). Patients with AEs were defined as experiencing the event at least once during the study treatment period, and all AEs were graded using Common Terminology Criteria for Adverse Events (CTCAE, version 3.0). The trial protocol and genetic biomarker studies were approved by the institutional review board at each site and were in accordance with the Declaration of Helsinki, US Food and Drug Administration Good Clinical Practices, and local ethics and legal requirements. All patients included in this study provided written informed consent for genetic biomarker testing. ClinicalTrials.gov NCT00851084.

Somatic mutation selection and profiling. Ninety-six hotspot mutations in eight genes frequently mutated in mCRC were selected from the COSMIC database (Supplementary Table S1). After the tumour area was marked by a pathologist, tumour DNA was extracted from three formalin-fixed paraffin-embedded tumour slides per patient collected at the time of diagnosis using the Qiagen DNAeasy kit (Qiagen, Antwerp, Belgium). Mutation profiling was carried out using Sequenom MassARRAY (Sequenom, San Diego, CA, USA) as previously described (Lambrechts et al, 2012; Zhao et al, 2014). Mutations were grouped by gene for the prediction of efficacy or safety.

SNP selection and genotyping. One hundred and eighty-three tagging SNPs in VEGF pathway genes (Lambrechts et al, 2012) and four SNPs known to be associated with an increased susceptibility to hypertension and thrombosis were selected (Supplementary Table S2). Germline DNA was extracted from peripheral blood using the Qiagen DNAeasy kit (Qiagen, Belgium) and genotyped using Sequenom MassARRAY as previously described (Zhao et al, 2014).

Plasma profiling. The plasma concentration of 27 cytokines, growth factors or soluble receptors was determined by enzymelinked immunosorbent assays (ELISA) using two Fluorokine MAP kits (the human angiogenesis panel $\mathrm{A}$ and the human high sensitivity cytokine panel; R\&D Systems, Abingdon, UK). Competition experiments were conducted to test interference of aflibercept with the detection of VEGFA, VEGFD and placental growth factor (PlGF). Angiopoietin-2 (ANGPT2), SDF1- $\alpha$, HGF, VEGFC, soluble VEGF receptor 3 (sVEGFR3) and sVEGFR2 were assessed by single ELISA (R\&D Systems). Plasma markers were analysed at baseline, at 30 and 60 days after the first study treatment infusion and 30 days after the last aflibercept infusion.

Statistical analysis. Differences between patients with evaluable biomarkers and patients without evaluable biomarkers were assessed using a two-sided Fisher's exact test for categorical variables and ANOVA for continuous variables. Biomarkers were analysed as quantitative variables, by coding the absence or presence of a somatic mutation as 0 or 1 , and SNP genotypes as 0 , 1 or 2 depending on the number of minor alleles present. The linear effects of baseline biomarkers on PFS were assessed using a Cox proportional hazard model with the following co-variates: Eastern Cooperative Oncology Group performance status (0-1 vs 2), liver-only metastases (yes/no), and the number of distant metastasis organs $(1 v s>1)$, a treatment effect, a biomarker effect and a biomarker-treatment interaction effect. The significance of the latter two effects was jointly tested by a twodegrees-of-freedom Wald test. Extended statistical methods are described in Supplementary Methods.

\section{RESULTS}

Of the 236 patients in the ITT population of the AFFIRM trial, 227 (96\%) were evaluable for PFS. Of these, 130 (57\%) provided at least one biological sample, 60 (46\%) and 70 (54\%) of which participated in the mFOLFOX6 and mFOLFOX6 plus aflibercept arms, respectively. There was no difference at a $P<0.05$ between patients who provided a biological sample and those who did not in terms of patient biometrics, ethnicity and disease characteristics at baseline, or at efficacy and safety end points (Table 1). Of those who provided at least one biological sample, 51 (39\%) provided samples for each of the three biomarker types, with $88(68 \%)$ and $97(74 \%)$ patients providing samples for two or one of the biomarker types, 
respectively. Each biomarker type was analysed separately, to avoid patient groups that were too small for sub-analyses.

Somatic mutations and efficacy. Somatic mutation profiling was performed on tumour DNA obtained from 93 out of 227 (41\%) patients evaluable for response, 47 (51\%) of whom had been treated with aflibercept. The median mutation call rate per patient was $96.4 \%$ and $89(96 \%)$ patients had a call rate $>80 \%$. Mutations were detected in KRAS $(n=31)$, PIK3CA $(n=10)$, PIK3R1 $(n=5)$, $\operatorname{BRAF}(n=3)$ and NRAS $(n=3) ; 45$ patients carried only wild-type (wt) alleles, 44 patients carried a single mutation and four carried a mutation both in PIK3CA and KRAS. We assessed the effect of the gene mutation status on PFS, while allowing for interaction with both treatment arms. $P$-values were lowest, but not significant for KRAS (unadjusted $P$-value $=0.0601$; multiple testing-adjusted false discovery rate $(F D R)=0.30$; Supplementary Table S3). Although patients carrying mutant KRAS tumours exhibited a slightly worse median PFS when treated with mFOLFOX6 compared with patients treated with mFOLFOX6 plus aflibercept (7.7 vs 10.1 months), this did not reach significance (Table 2). There was also

Table 1. Summary of baseline disease characteristics in patients from the biomarker evaluable or non-evaluable population

\begin{tabular}{|c|c|c|c|c|}
\hline & \multicolumn{2}{|c|}{ Non-evaluable population } & \multicolumn{2}{|c|}{ Evaluable population } \\
\hline & $\begin{array}{c}\text { mFOLFOX6 } \\
(N=57)\end{array}$ & $\begin{array}{c}\text { Aflibercept plus } \\
\text { mFOLFOX6 } \\
(N=49)\end{array}$ & $\begin{array}{c}\text { mFOLFOX6 } \\
(N=60)\end{array}$ & $\begin{array}{l}\text { Aflibercept plus } \\
\text { mFOLFOX6 } \\
(N=70)\end{array}$ \\
\hline Gender: male/female & $32 / 25$ & $33 / 16$ & $36 / 24$ & $43 / 27$ \\
\hline Age: median (min : max) & $66(44: 87)$ & $62(29: 75)$ & $62(37: 81)$ & $63(41: 79)$ \\
\hline Age: $<65 / \geqslant 65$ years & $27 / 30$ & $28 / 21$ & $38 / 22$ & $42 / 25$ \\
\hline Race: Caucasian/Asian/Black/other & $41 / 16 / 0 / 0$ & $37 / 11 / 0 / 1$ & $49 / 11 / 0 / 0$ & $60 / 9 / 1 / 0$ \\
\hline Weight (kg): median (min : max) & $68(40: 107)$ & $70(40: 115)$ & $73(48: 134)$ & $71(40: 117)$ \\
\hline BSA $\left(m^{2}\right):$ median ( $\left.\min : \max \right)$ & $1.8(1: 2)$ & $1.8(1: 2)$ & $1.8(1: 2)$ & $1.8(1: 2)$ \\
\hline Prior adjuvant chemotherapy: yes/no & $50 / 7$ & $45 / 4$ & $55 / 5$ & $63 / 7$ \\
\hline Prior surgery: yes/no & $35 / 22$ & $27 / 22$ & $26 / 34$ & $29 / 41$ \\
\hline Prior radiotherapy: yes/no & $53 / 4$ & $47 / 2$ & $57 / 3$ & $62 / 8$ \\
\hline ECOG performance status: 0/1/2 & $55 / 2$ & $46 / 3$ & $60 / 0$ & $69 / 1$ \\
\hline Number of metastatic organs involved at baseline: $0 / 1 />1$ & $0 / 16 / 41$ & $0 / 15 / 34$ & $1 / 15 / 44$ & $0 / 12 / 58$ \\
\hline Liver-only metastases: yes/no & $15 / 42$ & $20 / 29$ & $18 / 42$ & $14 / 56$ \\
\hline Abbreviation: ECOG = Eastern Cooperative Oncology Group. & & & & \\
\hline
\end{tabular}

Table 2. Kaplan-Meier estimates of effect of biomarkers on months of PFS

\begin{tabular}{|c|c|c|c|}
\hline & $\begin{array}{c}\text { mFOLFOX6 median } \\
(99 \% \mathrm{Cl})\end{array}$ & $\begin{array}{c}\text { Aflibercept plus mFOLFOX6 } \\
\text { median }(99 \% \mathrm{Cl})\end{array}$ & $\begin{array}{c}\text { Hazard ratio vs mFOLFOX6 } \\
(99 \% \mathrm{Cl})\end{array}$ \\
\hline \multicolumn{4}{|l|}{ Somatic mutations } \\
\hline All patients & $10.9(8.80-14.19)$ & $8.5(7.72-11.63)$ & $1.439(0.739-2.801)$ \\
\hline $\begin{array}{l}\text { KRAS wt } \\
\text { KRAS mt } \\
\text { RAS wt } \\
\text { RAS mt }\end{array}$ & $\begin{array}{l}11.2(7.62-12.48) \\
10.1(3.71-\mathrm{NC}) \\
10.0(7.62-12.19) \\
10.9(3.71-15.64)\end{array}$ & $\begin{array}{r}10.1(7.95-12.78) \\
7.7(5.82-12.85) \\
10.1(7.95-12.88) \\
7.9(6.67-10.48)\end{array}$ & $\begin{array}{l}0.971(0.424-2.221) \\
2.571(0.785-8.423) \\
0.888(0.362-2.176) \\
2.340(0.855-6.404)\end{array}$ \\
\hline \multicolumn{4}{|l|}{ Germline SNPs } \\
\hline All patients & $9.3(8.11-11.33)$ & $9.0(7.46-10.91)$ & $1.304(0.7-2.432)$ \\
\hline $\begin{array}{l}\text { rs3741403 } \\
C / C \\
C / T \text { or } T / T\end{array}$ & $\begin{array}{c}8.9(6.28-9.46) \\
10.9(7.10-14.23)\end{array}$ & $\begin{array}{l}6.7(3.81-9.99) \\
9.3(8.08-11.70)\end{array}$ & $\begin{array}{l}1.322(0.459-3.806) \\
1.412(0.667-2.989)\end{array}$ \\
\hline $\begin{array}{l}\text { rs2346176 } \\
\mathrm{C} / \mathrm{C} \\
\mathrm{C} / \mathrm{T} \text { or } \mathrm{T} / \mathrm{T}\end{array}$ & $\begin{array}{r}11.8(9.23-15.57) \\
8.8(5.59-10.91)\end{array}$ & $\begin{array}{c}7.7(4.14-9.20) \\
10.0(7.92-11.10)\end{array}$ & $\begin{array}{l}3.449(0.934-12.73) \\
0.974(0.479-1.979)\end{array}$ \\
\hline $\begin{array}{l}\text { rs } 4953344 \\
\mathrm{~T} / \mathrm{T} \\
\mathrm{C} / \mathrm{C} \text { or } \mathrm{C} / \mathrm{T}\end{array}$ & $\begin{array}{r}9.2(5.62-10.91) \\
11.8(8.11-15.64)\end{array}$ & $\begin{array}{l}9.3(7.98-11.63) \\
7.5(3.81-10.91)\end{array}$ & $\begin{array}{r}0.87(0.426-1.778) \\
2.912(0.919-9.226)\end{array}$ \\
\hline \begin{tabular}{|l}
$\mathrm{rs} 2881324$ \\
$\mathrm{~T} / \mathrm{T}$ \\
$\mathrm{G} / \mathrm{G}$ or $\mathrm{G} / \mathrm{T}$
\end{tabular} & $\begin{array}{r}11.8(5.09-15.64) \\
9.2(7.10-10.91)\end{array}$ & $\begin{array}{l}8.1(4.63-9.99) \\
9.3(7.46-11.10)\end{array}$ & $\begin{array}{l}2.159(0.679-6.863) \\
0.888(0.432-1.822)\end{array}$ \\
\hline \multicolumn{4}{|c|}{ Plasma protein markers, baseline } \\
\hline All patients & $8.8(6.57-10.02)$ & $8.5(6.67-10.05)$ & $0.979(0.505-1.897)$ \\
\hline $\begin{array}{l}\mathrm{IL} 8 \leq 19 \mathrm{pg} \mathrm{ml}^{-1} \\
\mathrm{IL}^{-1}>19 \mathrm{pg} \mathrm{ml}^{-1}\end{array}$ & $\begin{array}{l}8.8(5.62-10.91) \\
8.8(5.09-15.64)\end{array}$ & $\begin{array}{l}9.3(7.52-11.10) \\
4.1(2.33-8.54)\end{array}$ & $\begin{array}{r}0.764(0.363-1.607) \\
2.71(0.735-9.984)\end{array}$ \\
\hline
\end{tabular}


no effect of the RAS (KRAS, BRAF and NRAS) mutation status on PFS (Table 2).

Germline SNPs and efficacy. Germline DNA was available for 97 randomised patients, 53 of whom had been treated with aflibercept, and was genotyped for 133 SNPs.

Principle component analysis showed that genotype variance was partly explained by race (Supplementary Figure S1). As the proportion of Asian patients in AFFIRM was quite large (16 Asian vs 81 non-Asian patients), we first analysed SNP data without Asian patients, and subsequently carried out a sensitivity analysis by including them while stratifying for race. Among the 133 SNPs tested, there were four SNPs with a $P<0.05$ on PFS and a multiple testing-adjusted FDR of 0.22. One SNP was in VEGFB, which is inhibited by aflibercept (rs3741403, $P=0.0021$ ), whereas three other SNPs were located in EPAS1, (rs2346176, $P=0.0051$; rs4953344, $P=0.0065$; and rs2881324, $P=0.0066$; Supplementary Table S4), which codes for HIF-2alpha, a mediator of cellular response to hypoxia. These SNPs also exhibited the lowest $P$-values when including Asian patients (Supplementary Table S4).

The combined effects of individual SNPs in a given gene were explored in a gene-wise association analysis. Of the 17 genes tested, we observed a significant effect for VEGFB, $(P=0.0025$; multiple testing-adjusted FDR $=0.0423$; Supplementary Table S5), in which three SNPs were genotyped, whereas EPAS ranked second (multiple testing-adjusted FDR $=0.1205$ ). To assess which combination of SNPs accounted for the effect on PFS, we estimated haplotype frequencies consisting of rs3741403, rs1058735 and rs594942 (Supplementary Table S6). The most frequent haplotype in VEGFB, that is, the TCC haplotype, was selected in a stepwise Cox model. The hazard ratio for the TCC haplotype was 0.214 , but the interaction with treatment arm was not selected, suggesting that it had a prognostic effect.

Profiling of plasma markers for efficacy. Plasma levels of 27 markers were measured at different time points (i.e., at baseline (87 patients); 30 and 60 days after start of treatment (82 and 73 patients); and 30 days after the last treatment (56 patients; Supplementary Table S7)). We assessed the association of each plasma marker at baseline with PFS, while allowing for an interaction with treatment (Supplementary Table S8). The lowest $P$-value was obtained for IL8 ( $P=0.0221$; multiple testing-adjusted $\mathrm{FDR}=0.596$ and $P=0.0218$ for interaction) with a possible threshold effect at $19 \mathrm{pg} \mathrm{ml}^{-1}$ IL8 (Table 1; Supplementary Methods).

We also analysed whether treatment-related changes in plasma markers could predict aflibercept treatment outcome. The Cox model included the effect of baseline plasma levels and the difference in expression measured at baseline and the last time point before discontinuation, disease progression or death (i.e., at 30 days or 60 days of treatment), while allowing for interaction with the treatment arm. IL8 was the only marker with a significant effect of change from baseline on PFS $(P=0.0018$; multiple testingadjusted FDR $=0.0478$; Table 3). In particular, high baseline or post-baseline increased IL8 levels corresponded to a higher probability of disease progression at 12 months (Figure 1). A multivariate Cox model with lasso penalisation was carried out to check if additional markers in combination with IL8 marker would improve prediction of PFS. A maximum of likelihood was not reached by the cross-validation procedure ( $R$ Package penalised), which was interpreted as a failure of additional biomarkers to better predict PFS.

Interestingly, some cytokines showed significant expression changes after the start of treatment. Expression levels at day 30 after treatment start were compared to baseline, and at day 60 to day 30 after treatment start. The following significant changes were detected after FDR adjustment in the aflibercept/mFOLFOX6 arm (Table 4): a decrease in soluble VEGFR3, an increase in PlGF and a decrease in ANGPT2 (Figure 2; Supplementary Figure S2).
Table 3. Effect of plasma marker changes from baseline on PFS

\begin{tabular}{|c|c|c|c|c|}
\hline \multirow[b]{2}{*}{ Protein } & \multicolumn{4}{|c|}{$P$-values } \\
\hline & $\begin{array}{l}\text { Joint } \\
\text { effect }\end{array}$ & $\begin{array}{c}\text { Joint effect } \\
\text { (multiple } \\
\text { testing-adjusted } \\
\text { FDR) }\end{array}$ & $\begin{array}{l}\text { Change } \\
\text { from } \\
\text { baseline } \\
\text { effect }\end{array}$ & $\begin{array}{l}\text { Treatment by } \\
\text { Change from } \\
\text { baseline effect }\end{array}$ \\
\hline IL8 & 0.0018 & 0.0478 & 0.0006 & 0.2028 \\
\hline IL10 & 0.0342 & 0.4525 & 0.5214 & 0.8204 \\
\hline VEGFA & 0.0619 & 0.4525 & 0.0189 & 0.0704 \\
\hline CXCL12 & 0.0670 & 0.4525 & 0.1714 & 0.0318 \\
\hline CSF2 & 0.0855 & 0.4619 & 0.0266 & 0.1682 \\
\hline VEGFC & 0.1127 & 0.5072 & 0.0742 & 0.9355 \\
\hline IL5 & 0.1886 & 0.7275 & 0.0684 & 0.1657 \\
\hline Endostatin & 0.2418 & 0.7882 & 0.9988 & 0.2668 \\
\hline PDGFA & 0.3092 & 0.7882 & 0.1508 & 0.9922 \\
\hline TNF & 0.3209 & 0.7882 & 0.1770 & 0.1554 \\
\hline IL4 & 0.3211 & 0.7882 & 0.1938 & 0.7631 \\
\hline FGF2 & 0.3851 & 0.8191 & 0.6468 & 0.3957 \\
\hline sVEGFR3 & 0.3944 & 0.8191 & 0.2533 & 0.1729 \\
\hline THBS2 & 0.4677 & 0.8426 & 0.2387 & 0.6683 \\
\hline FGF1 & 0.4770 & 0.8426 & 0.4669 & 0.2242 \\
\hline PIGF & 0.4993 & 0.8426 & 0.2501 & 0.2755 \\
\hline ANGPT2 & 0.5638 & 0.8924 & 0.3373 & 0.8394 \\
\hline IL1B & 0.6261 & 0.8924 & 0.3345 & 0.5414 \\
\hline PDGFB & 0.6583 & 0.8924 & 0.3730 & 0.6009 \\
\hline IL12 & 0.6611 & 0.8924 & 0.7934 & 0.7744 \\
\hline IL2 & 0.7984 & 0.9482 & 0.9352 & 0.6027 \\
\hline sVEGFR2 & 0.8375 & 0.9482 & 0.6009 & 0.5613 \\
\hline HGF & 0.8383 & 0.9482 & 0.5804 & 0.5665 \\
\hline IFNG & 0.8559 & 0.9482 & 0.6923 & 0.9101 \\
\hline ANGPT1 & 0.8814 & 0.9482 & 0.6269 & 0.8538 \\
\hline VEGFD & 0.9131 & 0.9482 & 0.6838 & 0.7528 \\
\hline IL6 & 0.9963 & 0.9963 & 0.9893 & 0.9594 \\
\hline
\end{tabular}

Abbreviations: $F D R=$ false discovery rate; $P F S=$ progression-free survival. $P$-values (and FDR) of the joint effects of plasma marker and treatment by plasma marker interactions are shown. $P$-values for the change from baseline and the interaction are also presented.

Plasma markers correlated with safety end points. Finally, we also assessed which of these biomarkers correlated with any of the following binary safety end points (occurrence of an $\mathrm{AE}$ or not): hypertension, proteinuria grade $\geqslant 2$ (either derived from laboratory data or when nephrotic syndrome was reported as an AE), haemorrhage or venous thromboembolism. Patients in the biomarker evaluable population were exposed slightly longer to treatment than patients in the population without evaluable biomarkers (median number of cycles: $12 v s 9$ or 10), but there was no difference in exposure between both treatment arms in the biomarker evaluable population (Supplementary Table S9). The frequency of these safety end points did not differ significantly between the populations with and without evaluable biomarkers (Supplementary Table S10). No effect of any type of biomarker (gene mutation status, SNP genotypes, baseline plasma biomarker levels) on any $\mathrm{AE}$ was observed at a multiple testing-adjusted FDR level below 0.5 (Supplementary Table S11). For each plasma marker, we also defined the cutoff levels that maximise the interaction with treatment. The optimal cutoff was obtained for HGF, at a level of $1.43 \mathrm{pg} \mathrm{ml}^{-1}$ (71th percentile). Although hypertension was strongly associated with aflibercept treatment 

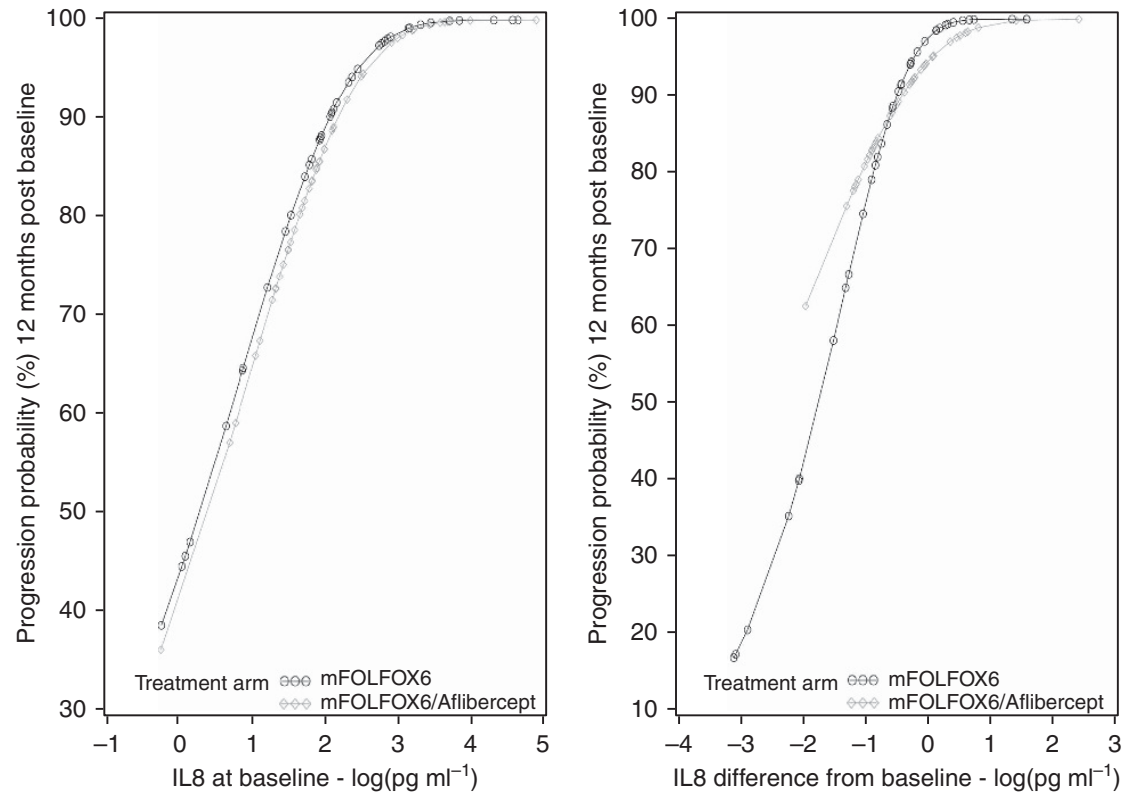

Figure 1. Relation between IL8 levels and probability of disease progression. Depicted is the probability of disease progression after 12 months in relation (left) to IL8 plasma levels at baseline and (right) to the difference between IL8 plasma levels at baseline and at the last measurement point before disease progression.

Table 4. Expression changes of plasma markers in response to treatment

\begin{tabular}{|l|l|c|c|c|}
\hline Plasma biomarker & Time-treatment & \multicolumn{1}{|c|}{$\begin{array}{c}\text { Slope estimate } \\
\text { (month }^{-1} \text { ) }\end{array}$} & $\begin{array}{c}\text { Multiple testing-adjusted } \\
\text { FDR }\end{array}$ \\
\hline sVEGFR3 & T1-aflibercept/mFOLFOX6 & -1.3619 & $<0.0001$ & $<0.0001$ \\
\hline PIGF & T1-aflibercept/mFOLFOX6 & 0.2081 & $<0.0001$ & $<0.0001$ \\
\hline HGF & T2-mFOLFOX6 & 0.1864 & 0.0004 & 0.0137 \\
\hline ANGPT2 & T1-aflibercept/mFOLFOX6 & -0.2722 & 0.0016 & 0.0443 \\
\hline
\end{tabular}

Abbreviation: FDR = false discovery rate. Table of slope estimates for expression changes of plasma markers (change from baseline) that are significantly different from zero. Slopes are estimated between baseline and day 30 (time T1), and between day 30 and day 60 (time T2) for each treatment arm.

$(\mathrm{OR}=50.4)$, only $20.0 \%$ of patients with high levels of plasma HGF $\left(>1.43 \mathrm{ng} \mathrm{ml}^{-1}\right)$ developed aflibercept-induced hypertension in the aflibercept/mFolfox6 treatment arm, compared with $75.8 \%$ of patients with low HGF levels $\left(<1.43 \mathrm{ng} \mathrm{ml}^{-1}\right.$; Supplementary Table S12).

\section{DISCUSSION}

AFFIRM was an open-label, non-comparative, phase II study conducted to assess the combination of aflibercept and mFOLFOX6 given as first-line therapy in patients with mCRC. Patients who received aflibercept in combination with mFOLFOX6 or mFOLFOX6 alone had 12-month PFS rates of 25.8\% (95\% CI: $17.2 \%-34.4 \%$ ) and $21.2 \%$ (95\% CI: $12.2 \%-30.3 \%$ ), respectively (Pericay et al, 2012). In contrast to bevacizumab, which prolongs PFS when combined with mFOLFOX6, aflibercept did not have a significant effect on PFS in this study. It should be noted, however, that in AFFIRM mFOLFOX6 arm only served as an internal benchmark, since the sample size of the study did not allow for an adequately powered statistical comparison between the two treatment groups. Nevertheless, this study provided an opportunity to conduct the first comprehensive biomarker analysis of aflibercept using blood and archived tumour tissue collected from participating patients. The most relevant finding is that treatment outcomes with aflibercept appear to be independent of (K)RAS mutation status. From a clinical standpoint, this observation is important as aflibercept is considered as a second-line treatment option for patients with mCRC previously treated with oxaliplatin (Bennouna et al, 2013). In first line, many patients may have been treated with the angiogenesis inhibitor bevacizumab combined with oxaliplatin (Van Cutsem et al, 2009). The OS and PFS outcomes of bevacizumab in the treatment of patients with mCRC are generally considered to be independent of KRAS gene status (Petrelli et al, 2013; Sastre et al, 2013). If the therapeutic effects of aflibercept had been limited to either KRAS wt or mutant tumours, this would have important consequences for second-line treatment options, as anti-EGFR treatments-which are generally used as a second- or third-line treatment after bevacizumab-are only recommended in KRAS wt tumours (Brodowicz et al, 2013). Our observations should be interpreted cautiously, however, as AFFIRM was a non-comparative study. Nevertheless, evaluation of our finding in further studies is warranted.

Studies suggest that chemokines and their receptors serve as important regulators of various metastatic and advanced cancers (Coussens and Werb, 2002; Miles et al, 2013). Interestingly, we identified that high IL8 levels at baseline correlated with shorter survival times, and patients with increasing levels of IL8 during treatment were more likely to progress. This suggests that patients with high IL8 levels, at baseline or during treatment, are at increased risk of disease progression during aflibercept therapy. 

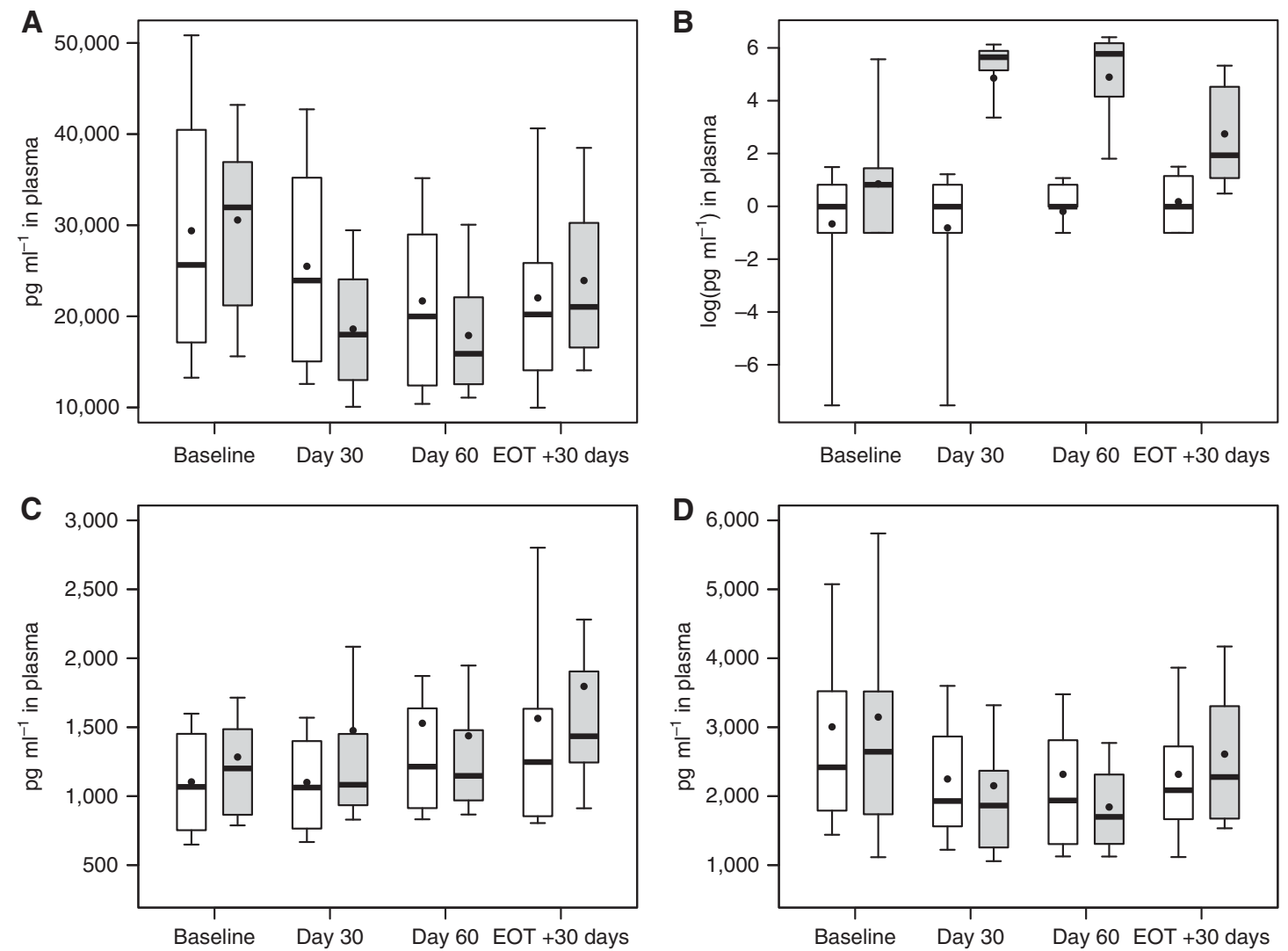

Figure 2. Plasma levels of sVEGFR3 (A), PIGF (B), HGF (C) and ANGPT2 (D). Concentrations are displayed for mFOLFOX6-treated (White) and mFOLFOX6/Aflibercept-treated (grey) patients at baseline, 30 days after the first study treatment infusion, 60 days after first infusion and 30 days after last infusion. Plasma levels for PIGF are log-transformed to allow visualisation of low levels of PIGF.

The role of IL8 and its receptor CXCR1 and -2 in tumour development and progression is well documented (Bennouna et al, 2013). Expression and secretion of IL8 by tumour cells enhances proliferation and survival through autocrine activation, and promotes angiogenesis and neutrophil infiltration into the tumour (Bennouna et al, 2013). IL8 also preserves the angiogenic response in HIF-1alpha-deficient colon cancer cells (Mizukami et al, 2005). Interestingly, in a phase II trial of 43 hepatocellular carcinoma patients receiving bevacizumab (Boige et al, 2012), and another phase II trial of $42 \mathrm{mCRC}$ patients on bevacizumab (Kopetz et al, 2010), elevated baseline IL8 also predicted shorter PFS times. Likewise, lower baseline IL8 levels were observed by Abajo et al (2012) in mCRC patients responding to a bevacizumab-containing therapy, whereas Hayashi et al (2014) observed increased baseline IL8 serum levels in non-responding mCRC patients participating to a phase 2 trial involving FOLFIRI chemotherapy plus bevacizumab. Similarly, IL8 levels correlated with OS in plasma samples from mCRC patients receiving capecitabine, oxaliplatin and bevacizumab (Liu et al, 2013). Another study in advanced hepatocellular carcinoma found that patients high levels of IL8 were also associated with poor outcome (Zhu et al, 2009), whereas in a phase 2 study in urotehlial cancer increases in IL8 after 4 weeks of pazopanib treatment were associated with a lower response probability (Necchi et al, 2014). SNPs in IL8 and its receptors have also been correlated with bevacizumab treatment outcome, thus further highlighting the potential role of IL8 in predicting response to anti-angiogenic therapies (Schultheis et al, 2008; Lambrechts et al, 2013).

In addition, we searched for relative changes in plasma markers induced by aflibercept treatment. We observed a decrease in sVEGFR3 and ANGPT2 levels, as well as an increase in PlGF levels. For PlGF, the increase most likely reflects an upregulation to compensate that most of the circulating PlGF is bound by aflibercept. We did not observe such compensatory upregulation for VEGFA. In this respect, aflibercept differs with respect to the consistent upregulation in VEGFA observed in most bevacizumab trials (Zhu et al, 2013). However, absence of a compensatory upregulation of VEGFA might be due to interference of aflibercept in the ELISA assay. Indeed, competition ELISA experiments for VEGFA, PIGF and VEGFD with aflibercept confirmed that aflibercept strongly interferes with VEGFA, but not PIGF or VEGFD antibodies used in the MAP kits (Supplementary Figure S3). In contrast to several other biomarker studies in mCRC involving bevacizumab treatment, we failed to see a change in sVEGFR2 following aflibercept treatment.

Finally, we also observed that ANGPT2 levels decrease following aflibercept delivery. ANGPT2 is a secreted factor that binds the endothelial cell-specific receptor tyrosine kinase TIE2 and has a complex role during angiogenesis. ANGPT2 is highly expressed by tumour endothelial cells, inhibits Tie2 activity and destabilises blood vessels, thereby facilitating VEGF-dependent vessel growth (Daly et al, 2013). Yet tumour xenografts treated with the combination of an ANGPT2-specific antibody (REGN910) and aflibercept demonstrated reduced tumour vascularity and tumour perfusion that was more pronounced than with either single agent, resulting in more extensive tumour cell death and more potent inhibition of tumour growth (Daly et al, 2013). Likewise, a human monoclonal anti-ANGPT2 antibody has a broader antitumor activity when combined with VEGF inhibitors (Brown et al, 2010). This indicates that ANGPT2 might indeed have a protective role in tumour endothelial cells by activating TIE2, thereby limiting the anti-vascular effects of VEGF inhibition. Interestingly, Goede et al (2010) reported that amongst $34 \mathrm{mCRC}$ patients receiving bevacizumab, low pre-therapeutic serum ANGPT2 levels were 
associated with a significant better response rate, prolonged median PFS and reduction of $91 \%$ in the risk of death. On the other hand, in tumour biopsies collected before and 12 days after bevacizumab monotherapy in rectal cancer patients, ANGPT1 and ANGPT2 were both downregulated in cancer cells. Our data therefore add to the emerging role of ANGPT2 as a potential mediator of response to anti-angiogenic therapies.

In conclusion, we here present the very first data investigating biomarkers for aflibercept treatment. Our observations for IL8, PlGF and ANGPT2 suggest that circulating cytokine levels before therapy, as well as their changes during aflibercept therapy, are consistent with those seen for other anti-VEGF agents. Pending validation in other aflibercept-containing regimens, these exploratory biomarker data could be useful to guide patient selection in future studies with aflibercept.

\section{ACKNOWLEDGEMENTS}

We would like to thank investigators, patients and their families. The manuscript was financially supported by Sanofi (Cambridge, MA). The Medical Affairs Department at Sanofi was allowed several courtesy reviews by the authors for scientific accuracy, and provided feedback to the authors for their consideration. Editorial assistance in the preparation of this manuscript was provided by Dr George Xinarianos (Cancer Communications Ltd, Knutsford, UK), and funded by Sanofi (Cambridge, MA). DL is supported by the EU's Framework Programme Seven (FP7) ANGIOPREDICT under contract \#306021.

\section{CONFLICT OF INTEREST}

DL and EVC have served on advisory boards for Sanofi and received research funding from Sanofi to conduct this study. GF has received honoraria for participation in ad hoc advisory boards and for lectures from Sanofi. JZ has received travel and research support and honoraria for participation in advisory boards from Sanofi. VT, CZ, EM and MC are employees of Sanofi. BT, MM, XS, $\mathrm{CP}$ and GP declare no conflict of interest. The clinical trial, collection of tissue for biomarker evaluation and the evaluation of biomarkers were sponsored and funded by Sanofi-Aventis, Paris, France. BT holds a postdoctoral fellowship of the Fund for Scientific Research Flanders (FWO-F).

\section{REFERENCES}

Abajo A, Boni V, Lopez I, Gonzalez-Huarriz M, Bitarte N, Rodriguez J, Zarate R, Bandres E, Garcia-Foncillas J (2012) Identification of predictive circulating biomarkers of bevacizumab-containing regimen efficacy in pretreated metastatic colorectal cancer patients. Br J Cancer 107(2): 287-290.

Bennouna J, Sastre J, Arnold D, Osterlund P, Greil R, Van Cutsem E, von Moos R, Vieitez JM, Bouche O, Borg C, Steffens CC, Alonso-Orduna V, Schlichting C, Reyes-Rivera I, Bendahmane B, Andre T, Kubicka S (2013) Continuation of bevacizumab after first progression in metastatic colorectal cancer (ML18147): a randomised phase 3 trial. Lancet Oncol 14(1): 29-37.

Boige V, Malka D, Bourredjem A, Dromain C, Baey C, Jacques N, Pignon JP, Vimond N, Bouvet-Forteau N, De Baere T, Ducreux M, Farace F (2012) Efficacy, safety, and biomarkers of single-agent bevacizumab therapy in patients with advanced hepatocellular carcinoma. Oncologist 17(8): 1063-1072. Brodowicz T, Ciuleanu TE, Radosavljevic D, Shacham-Shmueli E, Vrbanec D, Plate S, Mrsic-Krmpotic Z, Dank M, Purkalne G, Messinger D, Zielinski CC (2013) FOLFOX4 plus cetuximab administered weekly or every second week in the first-line treatment of patients with KRAS wild-type metastatic colorectal cancer: a randomized phase II CECOG study. Ann Oncol 24(7): 1769-1777.
Brown JL, Cao ZA, Pinzon-Ortiz M, Kendrew J, Reimer C, Wen S, Zhou JQ, Tabrizi M, Emery S, McDermott B, Pablo L, McCoon P, Bedian V, Blakey DC (2010) A human monoclonal anti-ANG2 antibody leads to broad antitumor activity in combination with VEGF inhibitors and chemotherapy agents in preclinical models. Mol Cancer Ther 9(1): $145-156$.

Chiron M, Bagley RG, Pollard J, Mankoo PK, Henry C, Vincent L, Geslin C, Baltes N, Bergstrom DA (2014) Differential antitumor activity of aflibercept and bevacizumab in patient-derived xenograft models of colorectal cancer. Mol Cancer Ther 13(6): 1636-1644.

Ciombor KK, Berlin J, Chan E (2013) Aflibercept. Clin Cancer Res 19(8): 1920-1925.

Coussens LM, Werb Z (2002) Inflammation and cancer. Nature 420(6917): 860-867.

Daly C, Eichten A, Castanaro C, Pasnikowski E, Adler A, Lalani AS, Papadopoulos N, Kyle AH, Minchinton AI, Yancopoulos GD, Thurston G (2013) Angiopoietin-2 functions as a Tie2 agonist in tumor models, where it limits the effects of VEGF inhibition. Cancer Res 73(1): $108-118$.

Goede V, Coutelle O, Neuneier J, Reinacher-Schick A, Schnell R, Koslowsky TC, Weihrauch MR, Cremer B, Kashkar H, Odenthal M, Augustin HG, Schmiegel W, Hallek M, Hacker UT (2010) Identification of serum angiopoietin-2 as a biomarker for clinical outcome of colorectal cancer patients treated with bevacizumab-containing therapy. Br J Cancer 103(9): 1407-1414.

Hayashi H, Arao T, Matsumoto K, Kimura H, Togashi Y, Hirashima Y, Horita Y, Iwasa S, Okita NT, Honma Y, Takashima A, Kato K, Hamaguchi T, Shimada Y, Nakagawa K, Nishio K, Yamada Y (2014) Biomarkers of reactive resistance and early disease progression during chemotherapy plus bevacizumab treatment for colorectal carcinoma. Oncotarget 5(9): 2588-2595.

Joulain F, Proskorovsky I, Allegra C, Tabernero J, Hoyle M, Iqbal SU, Van Cutsem E (2013) Mean overall survival gain with aflibercept plus FOLFIRI $v$ s placebo plus FOLFIRI in patients with previously treated metastatic colorectal cancer. Br J Cancer 109(7): 1735-1743.

Jubb AM, Harris AL (2010) Biomarkers to predict the clinical efficacy of bevacizumab in cancer. Lancet Oncol 11(12): 1172-1183.

Kopetz S, Hoff PM, Morris JS, Wolff RA, Eng C, Glover KY, Adinin R, Overman MJ, Valero V, Wen S, Lieu C, Yan S, Tran HT, Ellis LM, Abbruzzese JL, Heymach JV (2010) Phase II trial of infusional fluorouracil, irinotecan, and bevacizumab for metastatic colorectal cancer: efficacy and circulating angiogenic biomarkers associated with therapeutic resistance. J Clin Oncol 28(3): 453-459.

Lambrechts D, Claes B, Delmar P, Reumers J, Mazzone M, Yesilyurt BT, Devlieger R, Verslype C, Tejpar S, Wildiers H, de Haas S, Carmeliet P, Scherer SJ, Van Cutsem E (2012) VEGF pathway genetic variants as biomarkers of treatment outcome with bevacizumab: an analysis of data from the AViTA and AVOREN randomised trials. Lancet Oncol 13(7): 724-733.

Lambrechts D, Lenz HJ, de Haas S, Carmeliet P, Scherer SJ (2013) Markers of response for the antiangiogenic agent bevacizumab. J Clin Oncol 31(9): 1219-1230.

Lambrechts D, Moisse M, Delmar P, Miles DW, Leighl N, Escudier B, Van Cutsem E, Bansal AT, Carmeliet P, Scherer SJ, de Haas S, Pallaud C (2014) Genetic markers of bevacizumab-induced hypertension. Angiogenesis 17(3): 685-694.

Liu Y, Starr MD, Bulusu A, Pang H, Wong NS, Honeycutt W, Amara A, Hurwitz HI, Nixon AB (2013) Correlation of angiogenic biomarker signatures with clinical outcomes in metastatic colorectal cancer patients receiving capecitabine, oxaliplatin, and bevacizumab. Cancer Med 2(2): 234-242.

Miles DW, de Haas SL, Dirix LY, Romieu G, Chan A, Pivot X, Tomczak P, Provencher L, Cortes J, Delmar PR, Scherer SJ (2013) Biomarker results from the AVADO phase 3 trial of first-line bevacizumab plus docetaxel for HER2-negative metastatic breast cancer. Br J Cancer 108(5): 1052-1060.

Mizukami Y, Jo WS, Duerr EM, Gala M, Li J, Zhang X, Zimmer MA, Iliopoulos O, Zukerberg LR, Kohgo Y, Lynch MP, Rueda BR, Chung DC (2005) Induction of interleukin-8 preserves the angiogenic response in HIF-1alpha-deficient colon cancer cells. Nat Med 11(9): 992-997.

Necchi A, Pennati M, Zaffaroni N, Landoni E, Giannatempo P, Raggi D, Schwartz LH, Morosi C, Crippa F, Fare E, Nicolai N, Lanocita R, Sava T, Sacco C, Messina C, Ortega C, De Braud FG, Salvioni R, Daidone MG, Gianni AM, Mariani L (2014) Analysis of plasma cytokines and 
angiogenic factors in patients with pretreated urothelial cancer receiving Pazopanib: the role of circulating interleukin- 8 to enhance the prognostic accuracy. Br J Cancer 110(1): 26-33.

Pericay C, Folprecht G, Saunders M, Thomas A, Roh J, Lopez R (2012) Phase 2 randomized, noncomparative, open-label study of aflibercept and modified FOLFOX6 in the first-line treatment of metastatic colorectal cancer (AFFIRM). Ann Oncol 23(Suppl 4): iv16, Abstract O-0024.

Petrelli F, Coinu A, Cabiddu M, Ghilardi M, Barni S (2013) KRAS as prognostic biomarker in metastatic colorectal cancer patients treated with bevacizumab: a pooled analysis of 12 published trials. Med Oncol 30(3): 650.

Sastre J, Vidaurreta M, Gomez A, Rivera F, Massuti B, Lopez MR, Abad A, Gallen M, Benavides M, Aranda E, Rubio ED. Spanish Cooperative Group for the Treatment of Digestive T (2013) Prognostic value of the combination of circulating tumor cells plus KRAS in patients with metastatic colorectal cancer treated with chemotherapy plus bevacizumab. Clin Colorectal Cancer 12(4): 280-286.

Schneider BP, Li L, Shen F, Miller KD, Radovich M, O'Neill A, Gray RJ, Lane D, Flockhart DA, Jiang G, Wang Z, Lai D, Koller D, Pratt JH, Dang CT, Northfelt D, Perez EA, Shenkier T, Cobleigh M, Smith ML, Railey E, Partridge A, Gralow J, Sparano J, Davidson NE, Foroud T, Sledge GW (2014) Genetic variant predicts bevacizumab-induced hypertension in ECOG-5103 and ECOG-2100. Br J Cancer 111(6): 1241-1248.

Schultheis AM, Lurje G, Rhodes KE, Zhang W, Yang D, Garcia AA, Morgan R, Gandara D, Scudder S, Oza A, Hirte H, Fleming G, Roman L, Lenz HJ (2008) Polymorphisms and clinical outcome in recurrent ovarian cancer treated with cyclophosphamide and bevacizumab. Clin Cancer Res 14(22): 7554-7563.

Van Cutsem E, Rivera F, Berry S, Kretzschmar A, Michael M, DiBartolomeo M, Mazier MA, Canon JL, Georgoulias V, Peeters M, Bridgewater J, Cunningham D (2009) Safety and efficacy of first-line bevacizumab with
FOLFOX, XELOX, FOLFIRI and fluoropyrimidines in metastatic colorectal cancer: the BEAT study. Ann Oncol 20(11): 1842-1847.

Van Cutsem E, Tabernero J, Lakomy R, Prenen H, Prausova J, Macarulla T, Ruff P, van Hazel GA, Moiseyenko V, Ferry D, McKendrick J, Polikoff J, Tellier A, Castan R, Allegra C (2012) Addition of aflibercept to fluorouracil, leucovorin, and irinotecan improves survival in a phase III randomized trial in patients with metastatic colorectal cancer previously treated with an oxaliplatin-based regimen. J Clin Oncol 30(28): 3499-3506.

Zhao H, Thienpont B, Yesilyurt BT, Moisse M, Reumers J, Coenegrachts L, Sagaert X, Schrauwen S, Smeets D, Matthijs G, Aerts S, Cools J, Metcalf A, Spurdle A, ANECS, Amant F, Lambrechts D (2014) Mismatch repair deficiency endows tumors with a unique mutation signature and sensitivity to DNA double-strand breaks. Elife 3: e02725.

Zhu AX, Finn RS, Mulcahy M, Gurtler J, Sun W, Schwartz JD, Dalal RP, Joshi A, Hozak RR, Xu Y, Ancukiewicz M, Jain RK, Nugent FW, Duda DG, Stuart K (2013) A phase II and biomarker study of ramucirumab, a human monoclonal antibody targeting the VEGF receptor-2, as first-line monotherapy in patients with advanced hepatocellular cancer. Clin CancerRes 19(23): 6614-6623.

Zhu AX, Sahani DV, Duda DG, di Tomaso E, Ancukiewicz M, Catalano OA, Sindhwani V, Blaszkowsky LS, Yoon SS, Lahdenranta J, Bhargava P, Meyerhardt J, Clark JW, Kwak EL, Hezel AF, Miksad R, Abrams TA, Enzinger PC, Fuchs CS, Ryan DP, Jain RK (2009) Efficacy, safety, and potential biomarkers of sunitinib monotherapy in advanced hepatocellular carcinoma: a phase II study. J Clin Oncol 27(18): 3027-3035.

This work is published under the standard license to publish agreement. After 12 months the work will become freely available and the license terms will switch to a Creative Commons AttributionNonCommercial-Share Alike 4.0 Unported License.

Supplementary Information accompanies this paper on British Journal of Cancer website (http://www.nature.com/bjc) 